\title{
Unconventional Pressure Dependence of the Superfluid Density in the Nodeless Topological Superconductor $\alpha-\mathrm{PdBi}_{2}$
}

\author{
Debarchan Das $\odot,{ }^{1, *}$ Ritu Gupta, ${ }^{1}$ Christopher Baines, ${ }^{1}$ Hubertus Luetkens $\odot,{ }^{1}$ Dariusz Kaczorowski $\odot,{ }^{2}$ \\ Zurab Guguchia $\odot,{ }^{1}$ and Rustem Khasanov $\odot^{1, \dagger}$ \\ ${ }^{1}$ Laboratory for Muon Spin Spectroscopy, Paul Scherrer Institute, CH-5232 Villigen PSI, Switzerland \\ ${ }^{2}$ Institute of Low Temperature and Structure Research, Polish Academy of Sciences, Wroctaw, ul. Okólna 2, 50-422, Poland
}

(Received 10 June 2021; revised 18 October 2021; accepted 20 October 2021; published 16 November 2021)

\begin{abstract}
We investigated the superconducting properties of the topological superconductor $\alpha-\mathrm{PdBi}_{2}$ at ambient and external pressures up to $1.77 \mathrm{GPa}$ using muon spin rotation experiments. The ambient pressure measurements evince a fully gapped $s$-wave superconducting state in the bulk of the specimen. Alternating current magnetic susceptibility and muon spin rotation measurements manifest a continuous suppression of $T_{c}$ with increasing pressure. In parallel, we observed a significant decrease of superfluid density by $\sim 20 \%$ upon application of external pressure. Remarkably, the superfluid density follows a linear relation with $T_{c}$, which was found before in some unconventional topological superconductors and hole-doped cuprates. This finding signals a possible crossover from Bose-Einstein to Bardeen-Cooper-Schrieffer like condensation in $\alpha-\mathrm{PdBi}_{2}$.
\end{abstract}

DOI: $10.1103 /$ PhysRevLett.127.217002

Topological superconductors (TSCs) possess a full pairing gap in the bulk and gapless surface states protected by time-reversal symmetry (TRS) [1-4]. The general interest in searching for a novel TSC is associated with its intimate connection to Majorana bound states as low energy excitation $[4,5]$, which has potential applications in quantum computation [6,7]. Thus, TSC has attracted significant research interest recently due to its scientific importance and potential application prospects. Despite serious research efforts, the experimental realizations of bulk topological superconductivity in real materials remain considerably limited to a few classes of materials such as $\mathrm{Cu} / \mathrm{Sr} / \mathrm{Nb}$-doped $\mathrm{Bi}_{2} \mathrm{Se}_{3}$ [8-13], layered transition metal dichalcogenides [14-17], ternary transition metal pnictides $[18,19]$, In-doped SnTe [20], and a few half-Heusler compounds [21,22]. Interestingly, recent reports investigating various members of the Pd-Bi family have reinvigorated the interest in searching for potential TSCs in this class of materials. Because of its intrinsic capability to maintain strong spin orbit coupling, the Pd-Bi family turns out to be quite promising. For instance, noncentrosymmetric $\alpha$-PdBi with a superconducting transition at $T_{c}=3.8 \mathrm{~K}$ [23] exhibits Dirac cone electronic dispersion at $700 \mathrm{meV}$ [24,25] below the Fermi energy. Similarly, the compound $\beta-\mathrm{PdBi}_{2}$ is another member of the $\mathrm{Pd}-\mathrm{Bi}$ family that has been reported to possibly host a topological superconducting state below $T_{c}=5.3 \mathrm{~K}$ [26-28]. The Dirac point in this material is located at $2.41 \mathrm{eV}$ below the Fermi energy [29-32]. In this framework, the relatively new member of Pd-Bi family, $\alpha-\mathrm{PdBi}_{2}$, crystallizing in monoclinic centrosymmetric structure (space group $C 2 / \mathrm{m}$ ), turns out to be very promising due to its superconducting $\left(T_{c}=1.7 \mathrm{~K}\right)$ as well as nontrivial topological properties [33-35].

Recent angle-resolved photoemission spectroscopy (ARPES) results [35] on this compound reveal the presence of a Dirac point at $1.26 \mathrm{eV}$ below the Fermi energy at the zone center. Notably, the ARPES data also display multiple band crossings at the Fermi energy. This tempts us to speculate that the superconducting gap structure might consist of multiple gaps. However, the tunnel diode oscillator based penetration depth study [33] indicates an isotropic gap structure in this compound with the gap to $T_{c}$ ratio $\Delta(0) / k_{B} T_{c} \sim 2.0$. It must be noted that tunnel diode oscillator studies are very sensitive to surface superconductivity. Therefore, it is desired to probe the superconducting gap structure by means of a bulk experimental technique. Further, Zhou et al. [36] recently reported a very interesting pressure $(p)-T_{c}$ phase diagram in this compound manifesting a sudden increase in $T_{c}$ at $p=1.3 \mathrm{GPa}$. Interestingly, $\mathrm{x}$-ray studies under pressure reveal no structural change around this pressure. Thus, it is very important to understand the origin of the rise in $T_{c}$ and to check whether the superfluid density also shows such an enhancement with pressure. In this regard, muon spin rotation $(\mu \mathrm{SR})$ experiments at ambient and under different hydrostatic pressures are of great importance to address these aforementioned issues.

In this Letter, we report the results of our detailed $\mu \mathrm{SR}$ experiments on $\alpha-\mathrm{PdBi}_{2}$ at ambient and under different hydrostatic pressures. Ambient pressure measurements substantiate single-gap $s$-wave superconductivity with preserved TRS state in this compound. Furthermore, by performing ac-magnetic susceptibility (ACS) and $\mu \mathrm{SR}$ 
experiments under different hydrostatic pressures, we explored the pressure- $T_{c}$ phase diagram, which is noticeably different from the previous report by Zhou et al. [36]. Our results manifest a substantial and continuous decrease of superfluid density ( 20\%) and $T_{c}$ with application of pressure up to $1.77 \mathrm{GPa}$.

Pieces consisting of many single crystals (not cleaved) of $\alpha-\mathrm{PdBi}_{2}$ obtained using melt growth technique [33] were used for the present study. Ambient pressure and high pressure transverse-field (TF) $\mu \mathrm{SR}$ experiments were performed on HAL-9500 and GPD spectrometers at the Paul Scherrer Institute (Villigen, Switzerland), respectively. For pressure dependent measurements, we used a double-wall piston cylinder type of cell with an inner channel made of $\mathrm{CuBe}$ material, especially designed to perform $\mu \mathrm{SR}$ experiments under pressure [37-39], and Daphne oil (7373) as a pressure-transmitting medium. All TF experiments were done after a field-cooling procedure. The $\mu$ SR asymmetry spectra were analyzed using the open software package MUSRFIT [40].

Figure 1(a) depicts TF- $\mu$ SR asymmetry spectra for $\alpha-\mathrm{PdBi}_{2}$ at two selected temperatures above $(2.5 \mathrm{~K})$ and below $(0.08 \mathrm{~K}) T_{c}$, measured in an applied magnetic field of $10 \mathrm{mT}$. Above $T_{c}$, the spectra exhibits a very small relaxation due to the presence of random local fields associated with the nuclear magnetic moments. On the contrary, in the superconducting state, the formation of flux line lattice produces an inhomogeneous distribution of magnetic field [inset of Fig. 1 (b)], which increases the relaxation rate of the $\mu \mathrm{SR}$ signal. Figure 1(b) shows the normalized Fourier spectra for singlecrystal $\alpha-\mathrm{PdBi}_{2}$ at temperatures above $(2.5 \mathrm{~K})$ and below $(0.08 \mathrm{~K}) T_{c}$ obtained by means of the maximum entropy Fourier transform technique. While we observed a sharp peak in the Fourier amplitude around $10 \mathrm{mT}$ (external applied field) at $2.5 \mathrm{~K}$, confirming homogeneous field distribution throughout the sample, a fairly asymmetric broad signal was seen at $0.08 \mathrm{~K}$, evincing the fact that the sample is indeed in the superconducting mixed state. The formation of the flux line lattice causes this broadening of the line shape.

The temperature dependence of $\sigma_{\mathrm{sc}}(T)$ for $\alpha-\mathrm{PdBi}_{2}$ measured at an applied field of $\mu_{0} H=10 \mathrm{mT}$ is presented in Fig. 1(c). From the observed temperature dependence of $\sigma_{\mathrm{sc}}(T)$, the nature of the superconducting gap can be determined as $\sigma_{\mathrm{sc}}(T)$, which is proportional to the superfluid density. The solid red and blue lines in Fig. 1(c) represent fitting of the experimental data with isotropic $s$-wave and $d$-wave models [52-54], respectively (see the $\mathrm{SM}$ [41]). It is quite evident from the figure that the observed $\sigma_{\mathrm{sc}}(T)$ excludes the presence of any nodes and can be best described using a single isotropic gap ( $s$ wave) yielding a gap value of $\Delta(0)=0.18(1) \mathrm{meV}$ and $T_{c}=1.27(3)$. In this context, it is to be noted that the $T_{c}$ obtained from $\mu \mathrm{SR}$ measurements is slightly lower than that determined from heat capacity measurements. This is because of the fact that $\mu \mathrm{SR}$ measurements were carried out
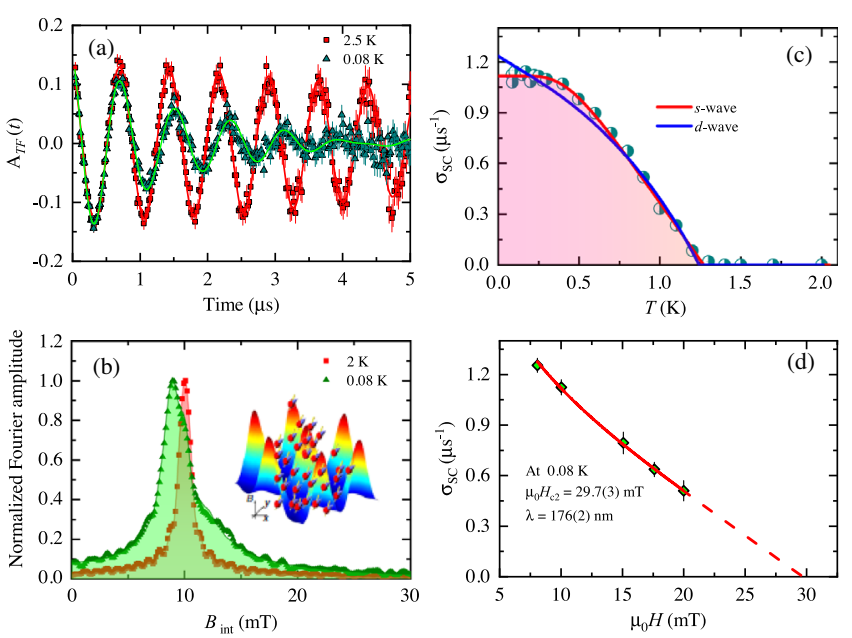

FIG. 1. (a) $\mathrm{TF} \mu \mathrm{SR}$ asymmetry spectra obtained above and below $T_{c}$ for $\alpha-\mathrm{PdBi}_{2}$. The spectra were recorded after field cooling the sample from above $T_{c}$ in an applied magnetic field of $\mu_{0} H=10 \mathrm{mT}$. Solid lines correspond to the fitting of the spectra as described in the Supplemental Material (SM) [41]. (b) Normalized Fourier spectra at $0.08 \mathrm{~K}$ (green) and $2 \mathrm{~K}$ (red) obtained by fast Fourier transformation of the spectra in (a). Inset: Schematic illustration of how muons, as local probes, sense the inhomogeneous magnetic field distribution in the vortex state of a type-II superconductor. (c) Temperature dependence of the superconducting muon spin depolarization rate $\sigma_{\mathrm{sc}}$ of $\alpha-\mathrm{PdBi}_{2}$ measured in an applied magnetic field of $\mu_{0} H=10 \mathrm{mT}$. The solid lines correspond to different theoretical models as discussed in the text. (d) Field dependence of $\sigma_{\text {sc }}$ fitted with the Brandt formula for an isotropic single $s$-wave gap (solid line) as discussed in the text.

in an applied field of $10 \mathrm{mT}$, which slightly lowered the superconducting transition temperature. From $\mu$ SR measurements we obtained the superconducting gap to $T_{c}$ ratio as $\Delta(0) / k_{B} T_{c}=1.64(1)$, which is very close to the value expected (1.764) for Bardeen-Cooper-Schrieffer (BCS) superconductors. It is worthwhile to emphasize that previous ARPES and band structure calculations [34,35] evince the presence of a Rashba state near the Fermi energy in this compound. It is theoretically predicted that in the presence of an in-plane magnetic field, the Majorana zero mode can be realized using the coupling of an $s$-wave superconductor with a material exhibiting Rashba states [55]. Thus, the observation of $s$-wave superconductivity in $\alpha-\mathrm{PdBi}_{2}$ is quite crucial in the search for Majorana fermions.

Furthermore, we also studied the field dependence of TF-relaxation rate $\sigma_{\mathrm{sc}}(B)$. Figure 1(d) illustrates the field dependence of $\sigma_{\mathrm{sc}}(B)$ at $0.08 \mathrm{~K}$. At each point, data were taken after field cooling the sample from $2 \mathrm{~K}$. We analyzed $\sigma_{\mathrm{sc}}(B)$ using the Brandt formula [56] for an single $s$ wave. Fitting with this model is shown by the red solid line in Fig. 1(d). From this analysis, we determined the values of upper critical field $\left[\mu_{0} H_{\mathrm{c} 2}(0)\right]$ and effective London penetration depth $\left(\lambda_{\text {eff }}\right)$ as 29.7(3) mT and 176(2) nm, respectively. Thus, the obtained value of $\mu_{0} H_{\mathrm{c} 2}(0)$ and $\lambda_{\mathrm{eff}}$ are in 
reasonably good agreement with the previous reports $[33,36] . \lambda_{\text {eff }}$ is related to the superconducting carrier density $n_{s}$ via the relation $\lambda_{\mathrm{eff}}^{2}=\left(m^{*} / \mu_{0} n_{s} e^{2}\right)$, where $m^{*}$ is the effective mass. Now $m^{*}$ can be estimated from the relation $m^{*}=\left(1+\lambda_{e-\mathrm{ph}}\right) m_{e}$ where $m_{e}$ is the electron rest mass and $\lambda_{e-\mathrm{ph}}$ is the electron-phonon coupling constant found out to be 0.54 from heat capacity (see the SM [41]). Thus, we estimated $n_{s}=1.41 \times 10^{27} \mathrm{~m}^{-3}$. This is comparable to the ones seen in some other TSCs, e.g., $\mathrm{Nb}_{0.25} \mathrm{Bi}_{2} \mathrm{Se}_{3}$ $\left(n_{s}=0.25 \times 10^{26} \mathrm{~m}^{-3}\right) \quad[13], \quad T_{d^{-}} \mathrm{MoTe}_{2} \quad\left(n_{s}=1.67 \times\right.$ $\left.10^{26} \mathrm{~m}^{-3}\right)$ [14], and 2M-WS ${ }_{2}\left(n_{s}=2.8 \times 10^{26} \mathrm{~m}^{-3}\right)$ [15]. Hall conductivity measurements estimating the carrier density in the normal state of this compound will be quite informative as they will allow us to compare the carrier densities in the normal and superconducting states. This will also provide information on whether the single-gap superconductivity in $\alpha-\mathrm{PdBi}_{2}$ originates from the superconducting gap occurring only on the electron or holelike Fermi surface. Moreover, results of our zero-field (ZF) $\mu$ SR measurements (not shown) confirm that the TRS state is preserved in the superconducting state of $\alpha-\mathrm{PdBi}_{2}$.

Figures 2(a) and 2(b) show the real $\left(\chi^{\prime}\right)$ and imaginary $\left(\chi^{\prime \prime}\right)$ components of the magnetic ACS of $\alpha-\mathrm{PdBi}_{2}$. The data in Figs. 2(a) and 2(b) are shifted vertically for better visualization. In the superconducting state, we see a clear drop in $\chi^{\prime}$ and a pronounced peak in $\chi^{\prime \prime}$. For constructing the pressure $(p)-T_{c}$ phase diagram from ACS measurements [see Fig. 3(a)], we considered the position of the peak in $\chi^{\prime \prime}$ [Fig. 2(b)] as $T_{c}$. It is quite evident that up to $1.77 \mathrm{GPa}, T_{c}$ decreases with increasing pressure. A linear fit to $T_{c}(p)$ [blue solid line in Fig. 3(a)] yields a slope $\left.\left(\delta T_{c} / \delta p\right)\right|_{\mathrm{ACS}}=-0.10(3) \mathrm{K} / \mathrm{GPa}$. This observation is in contrast to that previously reported by Zhou et al. [36], where $T_{c}$ showed a sudden jump with increasing pressure. It is important to note that for their transport measurements, Zhou et al. used $\mathrm{NaCl}$ powder as the pressure transmitting medium, which does not guarantee proper hydrostatic conditions. We speculate that such nonhydrostatic conditions can generate unexpectedly high pressures in micro grains of the sample resulting in local structural transition to $\beta-\mathrm{PdBi}_{2}$ phase. This might have increased the $T_{c}$ locally. As electrical transport measurements are sensitive to the filamentary superconducting channels, the enhancement in $T_{c}$ with increasing pressure as reported by Zhou et al. [36] might be an artifact of nonhydrostatic condition. On the other hand, as discussed the SM [41], all of our measurements were performed under hydrostatic conditions.

The TF- $\mu$ SR asymmetry spectra obtained from GPD experiments were analyzed as per the formalism presented in Ref. [39]. In Fig. 3(b), we present the temperature dependence of $\sigma_{\mathrm{sc}}(p, T)$ under various hydrostatic pressures, after normalizing it to the zero temperature value at ambient pressure [i.e., $\sigma_{\mathrm{sc}}(p=0, T=0)$ ]. As shown by the solid lines in Fig. 3(b), a single-gap $s$-wave model describes the $\sigma_{\mathrm{sc}}(T)$ data very well up to the highest pressure investigated. The pressure evolution of $T_{c}$ determined from $\mu \mathrm{SR}$
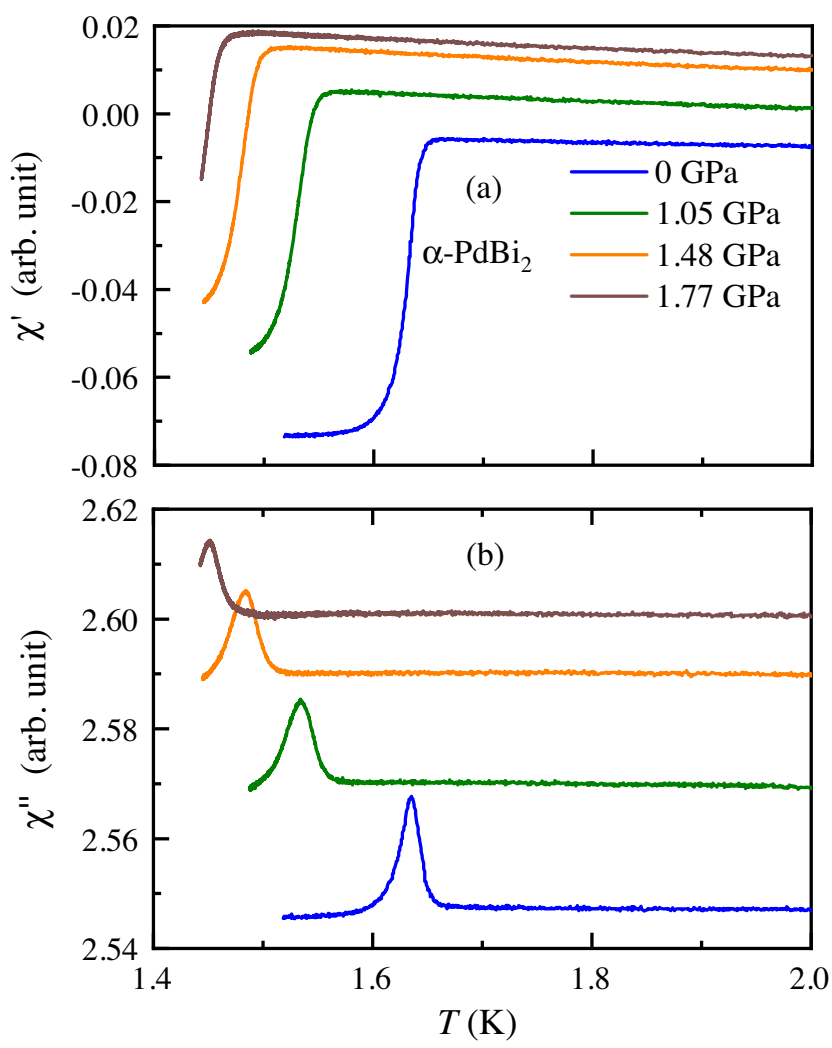

FIG. 2. Temperature dependence of the real, $\chi^{\prime}$, (a) and imaginary, $\chi^{\prime \prime}$, (b) components of ACS of $\alpha-\mathrm{PdBi}_{2}$ (mounted inside a double-wall piston cylinder type pressure cell made of $\mathrm{CuBe} / \mathrm{MP} 35 \mathrm{~N}$ material) at different selected pressures. For better visualization, we vertically shifted the $y$ axis in both (a) and (b).

experiments is shown in Fig 3(a). We note that the slope $\left.\left(\delta T_{c} / \delta p\right)\right|_{\mu \mathrm{SR}}=-0.11(1) \mathrm{K} / \mathrm{GPa}$ is very close to that determined from ACS measurements. The inset of Fig 3(a) shows the pressure variation of the gap value normalized to its zero pressure value, $\Delta_{0}(p) / \Delta_{0}(p=0)$, which also decreases with increasing pressure.

Remarkably, the ratio $\left[\sigma_{\mathrm{sc}}(p, T=0) / \sigma_{\mathrm{sc}}(p=0, T=0)\right]$, which is a direct measure of relative change of the superfluid density, is decreased considerably by $\sim 20 \%$ at 1.77 GPa. Such a significant reduction in superfluid density clearly indicates unconventional superconductivity in $\alpha-\mathrm{PdBi}_{2}$ as in conventional BCS superconductors the superfluid density either exhibits very weak pressure dependency or remains independent of pressure as highlighted by the horizontal dashed line in Fig. 4(a). Figure 4(a) shows the relative change of superfluid density and $T_{c}$ at different applied pressures presented in a color contour plot. This plot allows us to directly visualize the influence of pressure on these superconducting parameters and make a comparison with other known superconductors [14,16,57-62]. Notably, in the case of $\alpha-\mathrm{PdBi}_{2}$, we observe a clear deviation from the horizontal line that accounts for the BCS superconductors. For comparison, we included a few examples of unconventional superconductors that also 

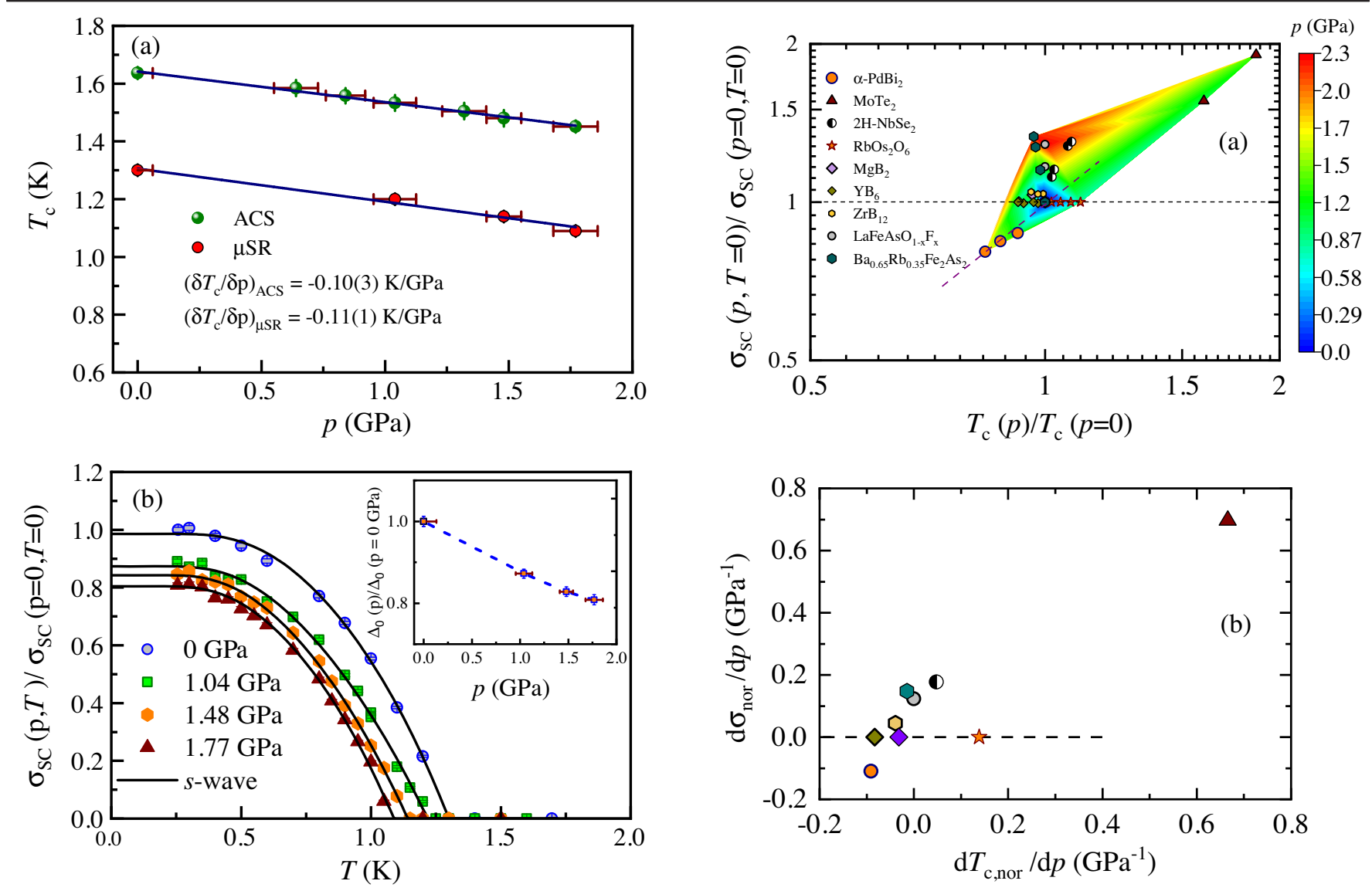

FIG. 3. (a) Pressure $(p)-T_{c}$ phase diagram of $\alpha-\mathrm{PdBi}_{2}$ constructed from ACS (green) and $\mu \mathrm{SR}$ (red) measurements under various applied hydrostatic pressures. Solid lines represent the linear fit through the experimentally obtained data points. Note that $T_{c}$ 's from $\mu \mathrm{SR}$ measurement are lower than those obtained from ACS because we performed the $\mu$ SR experiments under an applied field of $10 \mathrm{mT}$. (b) The temperature dependence of $\sigma_{\mathrm{sc}}(T)$ (normalized to its value at the zero applied pressure) under different applied pressures. Inset shows the pressure dependence of the superconducting gap $\Delta_{0}$ normalized to the zero pressure value.

demonstrate a clear deviation from the horizontal line. For better visualization, we have also presented $\mathrm{d} \sigma_{\text {nor }} / \mathrm{d} p$ as a function of $\mathrm{d} T_{c, \text { nor }} / \mathrm{d} p$ in Fig. 4(b), which manifests that the data points corresponding to the unconventional superconductors situated away from the horizontal line. Moreover, we observed a linear dependence between $T_{c}$ and the superfluid density for $\alpha-\mathrm{PdBi}_{2}$ [purple dashed line in Fig. 4(a)]. Similar linear dependence was also observed in the case of another TSC: $\mathrm{MoTe}_{2}$. In this context, it should be emphasized that the nearly linear relationship was originally observed in hole-doped cuprates in the underdoped region of the phase diagram [63,64]. Such linear relationship is expected only in the Bose-Einstein condensation (BEC)-like picture $[63,65,66]$. However, in the case of cuprates, the ratio between $T_{c}$ and their effective Fermi temperature $T_{F}$ is about $T_{c} / T_{F} \sim 0.05$, which

FIG. 4. (a) Normalized superfluid density as a function of $T_{c}(p) / T_{c}(p=0)$ at different applied pressures shown in a color contour plot. We compared the pressure effect with a few other conventional and unconventional superconductors. Black dashed horizontal line manifests the BCS prediction. Purple dashed line indicates the deviation from the BCS prediction and highlights the linear dependence between superfluid density and $T_{c}$ in $\alpha-\mathrm{PdBi}_{2}$. (b) $\mathrm{d} \sigma_{\text {nor }} / \mathrm{d} p$ as a function of $\mathrm{d} T_{c \text {,nor }} / \mathrm{d} p$ (where $X_{\text {nor }}=\left[\left(X_{p}-X_{p=0}\right) / X_{p=0}\right]$, with $X$ being $T_{c}$ or $\sigma_{\mathrm{sc}}$ for different superconductors depicted in panel (a).

suggests about a 4 to 5 times reduction of $T_{c}$ from the ideal BEC temperature for noninteracting Bose gas. These results were discussed in terms of the crossover from BEC to BCS-like condensation. Following the same line of reasoning, we anticipate a similar mechanism in $\alpha-\mathrm{PdBi}_{2}$.

In unconventional superconductors, one of the major factors determining $T_{c}$ is the superfluid density. Considering the linear dependency between $T_{c}$ and superfluid density, we speculate that the suppression of $T_{c}$ is a result of a reduction of superfluid density in this compound. Another possibility to observe negative pressure effect on $T_{c}$ is related to any weakening of the anharmonic electronlattice interactions with increasing pressure [15]. However, this picture cannot explain the substantial $(\sim 20 \%)$ reduction of superfluid density in $\alpha-\mathrm{PdBi}_{2}$. Nevertheless, pressure dependent detailed density functional calculations will be crucial to address this conjecture. 
The pressure effect on the superconducting properties of $\alpha-\mathrm{PdBi}_{2}$ reported by Zhou et al. [36] was found to be irreversible. Therefore, it seemed very important to check whether the effect observed by us in $\alpha-\mathrm{PdBi}_{2}$ was reversible or irreversible. We note that after releasing the pressure, we performed a quick temperature dependent TF- $\mu$ SR measurement that revealed that $\sigma_{\mathrm{sc}}(T)$ overlaps with that observed before applying any pressure (for details, see the SM [41]). This clearly suggests that the changes in superconducting properties seen here up to $1.77 \mathrm{GPa}$ are completely reversible.

In summary, we have performed $\mathrm{TF}$ and $\mathrm{ZF}-\mu \mathrm{SR}$ measurements on a topological superconductor candidate $\alpha-\mathrm{PdBi}_{2}$. The TF- $\mu \mathrm{SR}$ results reveal the absence of any nodes in the superconducting gap structure of this compound, and it can be best described by a fully gapped $s$-wave model. Observation of $s$-wave superconductivity in this system along with its unique band structure and Fermi surface topology, as demonstrated previously, endorse this compound as a promising candidate in the search for Majorana zero modes. Further, our ZF- $\mu$ SR study manifests that time reversal symmetry is preserved in the superconducting state of $\alpha-\mathrm{PdBi}_{2}$. Interestingly, ACS and TF- $\mu \mathrm{SR}$ experiments under different hydrostatic pressures reveal a negative pressure effect on $T_{c}$, which is suppressed at an rate of $-0.10 \mathrm{k} / \mathrm{GPa}$, upon application of pressure up to 1.77 GPa. Notably, the superconducting gap value and the superfluid density also decrease with increasing pressure up to $1.77 \mathrm{GPa}$. The most intriguing observation of the present study is the linear dependence between $T_{c}$ and the superfluid density manifesting in unconventional superconductivity in this compound. This also signals possible crossover from BEC to BCS in this compound. Further experimental and theoretical works under hydrostatic pressure conditions are called for to fully comprehend the pressure dependent phase diagram of $\alpha-\mathrm{PdBi}_{2}$. Our results on $\alpha-\mathrm{PdBi}_{2}$ indicating a substantial content of novel physics in this compound open more prospects for studies on TSC and its potential application.

This work was performed at the Swiss Muon Source $(\mathrm{S} \mu \mathrm{S})$ Paul Scherrer Institute, Villigen, Switzerland. The work of R. G. was supported by the Swiss National Science Foundation (SNF Grant No. 200021-175935). D. K. was supported by the National Science Centre (Poland) under research Grant No. 2015/18/A/ST3/00057.

*Corresponding author.

debarchandas.phy@gmail.com

${ }^{\dagger}$ Corresponding author. rustem.khasanov@psi.ch

[1] X.-L. Qi and S.-C. Zhang, Rev. Mod. Phys. 83, 1057 (2011).

[2] Y. Ando and L. Fu, Annu. Rev. Condens. Matter Phys. 6, 361 (2015).

[3] M. Sato and Y. Ando, Rep. Prog. Phys. 80, 076501 (2017).
[4] M. Z. Hasan and C. L. Kane, Rev. Mod. Phys. 82, 3045 (2010).

[5] F. Wilczek, Nat. Phys. 5, 614 (2009).

[6] A. Y. Kitaev, Phys. Usp. 44, 131 (2001).

[7] D. A. Ivanov, Phys. Rev. Lett. 86, 268 (2001).

[8] Y. S. Hor, A. J. Williams, J. G. Checkelsky, P. Roushan, J. Seo, Q. Xu, H. W. Zandbergen, A. Yazdani, N. P. Ong, and R. J. Cava, Phys. Rev. Lett. 104, 057001 (2010).

[9] M. Kriener, K. Segawa, Z. Ren, S. Sasaki, and Y. Ando, Phys. Rev. Lett. 106, 127004 (2011).

[10] J. A. Krieger, A. Pertsova, S. R. Giblin, M. Dobeli, T. Prokscha, C. W. Schneider, A. Suter, T. Hesjedal, A. V. Balatsky, and Z. Salman, Phys. Rev. Lett. 125, 026802 (2020).

[11] M. P. Smylie, H. Claus, U. Welp, W.-K. Kwok, Y. Qiu, Y. S. Hor, and A. Snezhko, Phys. Rev. B 94, 180510(R) (2016).

[12] K. Kobayashi, T. Ueno, H. Fujiwara, T. Yokoya, and J. Akimitsu, Phys. Rev. B 95, 180503(R) (2017).

[13] D. Das, K. Kobayashi, M. P. Smylie, C. Mielke, III, T. Takahashi, K. Willa, J.-X. Yin, U. Welp, M. Z. Hasan, A. Amato, H. Luetkens, and Z. Guguchia, Phys. Rev. B 102, 134514 (2020).

[14] Z. Guguchia et al., Nat. Commun. 8, 1082 (2017).

[15] Z. Guguchia, D. J. Gawryluk, M. Brzezinska, S. S. Tsirkin, R. Khasanov, E. Pomjakushina, F. O. von Rohr, J. A. T. Verezhak, M. Z. Hasan, T. Neupert, H. Luetkens, and Alex Amato, npj Quantum Mater. 4, 50 (2019).

[16] F. O. von Rohr, J.-C. Orain, R. Khasanov, C. Witteveen, Z. Shermadini, A. Nikitin, J. Chang, A. R. Wieteska, A. N. Pasupathy, M. Z. Hasan, A. Amato, H. Luetkens, Y. J. Uemura, and Z. Guguchia, Sci. Adv. 5, eaav8465 (2019).

[17] H.-J. Noh, J. Jeong, E.-J. Cho, K. Kim, B. I. Min, and B.-G. Park, Phys. Rev. Lett. 119, 016401 (2017).

[18] Y. Qian, S. Nie, C. Yi, L. Kong, C. Fang, T. Qian, H. Ding, Y. Shi, Z. Wang, H. Weng, and Z. Fang, npj Comput. Mater. 5, 121 (2019).

[19] D. Das, D. T. Adroja, M. R. Lees, R. W. Taylor, Z. S. Bishnoi, V. K. Anand, A. Bhattacharyya, Z. Guguchia, C. Baines, H. Luetkens, G. B. G. Stenning, L. Duan, X. Wang, and C. Jin, Phys. Rev. B 103, 144516 (2021).

[20] S. Sasaki, Z. Ren, A. A. Taskin, K. Segawa, L. Fu, and Y. Ando, Phys. Rev. Lett. 109, 217004 (2012).

[21] S. Chadov, X. Qi, J. Kübler, G. H. Fecher, C. Felser, and S. C. Zhang, Nat. Mater. 9, 541 (2010).

[22] H. Lin, L. A. Wray, Y. Xia, S. Xu, S. Jia, R. J. Cava, A. Bansil, and M. Z. Hasan, Nat. Mater. 9, 546 (2010).

[23] B. Joshi, A. Thamizhavel, and S. Ramakrishnan, Phys. Rev. B 84, 064518 (2011).

[24] Z. Sun, M. Enayat, A. Maldonado, C. Lithgow, E. Yelland, D. C. Peets, A. Yaresko, A. P. Schnyder, and P. Wahl, Nat. Commun. 6, 6633 (2015).

[25] M. Neupane, N. Alidoust, M. M. Hosen, J.-Xin Zhu, K. Dimitri, S.-Yang Xu, N. Dhakal, R. Sankar, I. Belopolski, D. S. Sanchez, T.-Rong Chang, H.-Tay Jeng, K. Miyamoto, T. Okuda, H. Lin, A. Bansil, D. Kaczorowski, F. Chou, M. Z. Hasan, and T. Durakiewicz, Nat. Commun. 7, 13315 (2016).

[26] Y. Imai, F. Nabeshima, T. Yoshinaka, K. Miyatani, R. Kondo, S. Komiya, I. Tsukada, and A. Maeda, J. Phys. Soc. Jpn. 81, 113708 (2012). 
[27] P. K. Biswas, D. G. Mazzone, R. Sibille, E. Pomjakushina, K. Conder, H. Luetkens, C. Baines, J. L. Gavilano, M. Kenzelmann, A. Amato, and E. Morenzoni, Phys. Rev. B 93, 220504(R) (2016).

[28] J. Kačmarčík, Z. Pribulová, T. Samuely, P. Szabó, V. Cambel, J. Šoltýs, E. Herrera, H. Suderow, A. CorreaOrellana, D. Prabhakaran, and P. Samuely, Phys. Rev. B 93, 144502 (2016).

[29] M. Sakano, K. Okawa, M. Kanou, H. Sanjo, T. Okuda, T. Sasagawa, and K Ishizaka, Nat. Commun. 6, 8595 (2015).

[30] K. Iwaya, Y. Kohsaka, K. Okawa, T. Machida, M. S. Bahramy, T. Hanaguri, and T. Sasagawa, Nat. Commun. 8, 976 (2017).

[31] A. Kolapo, T. Li, P. Hosur, and J. H. Miller, Jr., Sci. Rep. 9 , 12504 (2019).

[32] J. Chen, A. Wang, G.Pang, H. Su, Y. Chen, and H. Yuan, Phys. Rev. B 101, 054514 (2020).

[33] S. Mitra, K. Okawa, S. Kunniniyil Sudheesh, T. Sasagawa, Jian-Xin Zhu, and Elbert E. M. Chia, Phys. Rev. B 95, 134519 (2017).

[34] H. Choi, M. Neupane, T. Sasagawa, E. E. M. Chia, and J.-X. Zhu, Phys. Rev. Mater. 1, 034201 (2017).

[35] K. Dimitri, M. M. Hosen, G. Dhakal, H. Choi, F. Kabir, C. Sims, D. Kaczorowski, T. Durakiewicz, J.-Xin Zhu, and M. Neupane, Phys. Rev. B 97, 144514 (2018).

[36] Y. Zhou, X. Chen, C. An, Y. Zhou, L. Ling, J. Yang, C. Chen, L. Zhang, M. Tian, Z. Zhang, and Z. Yang, Phys. Rev. B 99, 054501 (2019).

[37] D. Andreica, Magnetic phase diagram in some Kondolattice compounds, Ph.D. thesis, ETH Zurich, 2001.

[38] R. Khasanov, Z. Guguchia, A. Maisuradze, D. Andreica, M. Elender, A. Raselli, Z. Shermadini, T. Goko, E. Morenzoni, and A. Amato, High Press. Res. 36, 140 (2016).

[39] Z. Shermadini, R. Khasanov, M. Elender, G. Simutis, Z Guguchia, K. V. Kamenev, and A Amato, High Press. Res. 37, 449 (2017).

[40] A. Suter and B. Wojek, Phys. Procedia 30, 69 (2012).

[41] See Supplemental Material, which includes Refs. [42-51], at http://link.aps.org/supplemental/10.1103/PhysRevLett .127 .217002 for (i) information related to the sample preparation and characterization, (ii) analysis of the $\mu \mathrm{SR}$ data, and (iii) reversibility check of the pressure effects in $\alpha-\mathrm{PdBi}_{2}$.

[42] D. Das, R. Gupta, A. Bhattacharyya, P. K. Biswas, D. T. Adroja, and Z. Hossain, Phys. Rev. B 97, 184509 (2018).

[43] L. Murata, H. Yoshino, H. O. Yadav, Y. Honda, and N. Shirakawa, Rev. Sci. Instrum. 68, 2490 (1997).

[44] M. H. Fang, H. M. Pham, B. Qian, T. J. Liu, E. K. Vehstedt, Y. Liu, L. Spinu, and Z. Q. Mao, Phys. Rev. B 78, 224503 (2008).
[45] R. Khasanov, D. G. Eshchenko, D. Di Castro, A. Shengelaya, F. La Mattina, A. Maisuradze, C. Baines, H. Luetkens, J. Karpinski, S. M. Kazakov, and H. Keller, Phys. Rev. B 72, 104504 (2005).

[46] C. Kittel, Introduction to Solid State Physics, 8th ed. (Wiley, New York, 2005).

[47] W. L. McMillan, Phys. Rev. 167, 331 (1968).

[48] A. M. Vora, Phys. Scr. 76, 204 (2007).

[49] K. Domieracki and D. Kaczorowski, J. Alloy Compd. 688, 206 (2016).

[50] G. Anemone, P. C. Aguilar, M. Garnica, F. Calleja, A. Al Taleb, C.-N Kuo, C. S. Lue, A. Politano, A. L. Vázquez de Parga, Giorgio Benedek, Daniel Farías, and R. Miranda, NPJ 2D Mater. Appl. 5, 25 (2021).

[51] R. Sharma, S. Dwivedi, and Y. Sharma, AIP Conf. Proc. 1665, 130003 (2015).

[52] M. Tinkham, Introduction to Superconductivity, 2nd ed. (Dover, Mineola, New York, 1996).

[53] R. Prozorov and R. W. Giannetta, Supercond. Sci. Technol. 19, R41 (2006).

[54] A. Carrington and F. Manzano, Physica (Amsterdam) 385C, 205 (2003).

[55] A. C. Potter and P. A. Lee, Phys. Rev. B 85, 094516 (2012).

[56] E. H. Brandt, Phys. Rev. B 37, 2349 (1988).

[57] D. Di Castro, R. Khasanov, C. Grimaldi, J. Karpinski, S. M. Kazakov, R. Brütsch, and H. Keller, Phys. Rev. B 72, 094504 (2005).

[58] R. Khasanov, D. G. Eshchenko, J. Karpinski, S. M. Kazakov, N. D. Zhigadlo, R. Brütsch, D. Gavillet, D. Di Castro, A. Shengelaya, F. La Mattina, A. Maisuradze, C. Baines, and H. Keller, Phys. Rev. Lett. 93, 157004 (2004).

[59] R. Khasanov, D. Di Castro, M. Belogolovskii, Yu. Paderno, V. Filippov, R. Brütsch, and H. Keller, Phys. Rev. B 72, 224509 (2005).

[60] R. Khasanov, P. S. Häfliger, N. Shitsevalova, A. Dukhnenko, R. Brütsch, and H. Keller, Phys. Rev. Lett. 97, 157002 (2006).

[61] G. Prando, Th. Hartmann, W. Schottenhamel, Z. Guguchia, S. Sanna, F. Ahn, I. Nekrasov, C. G. F. Blum, A. U. B. Wolter, S. Wurmehl, R. Khasanov, I. Eremin, and B. Büchner, Phys. Rev. Lett. 114, 247004 (2015).

[62] Z. Guguchia, A. Amato, J. Kang, H. Luetkens, P. K. Biswas, G. Prando, F. von Rohr, Z. Bukowski, A. Shengelaya, H. Keller, E. Morenzoni, Rafael M. Fernandes, and R. Khasanov, Nat. Commun. 6, 8863 (2015).

[63] Y. J. Uemura, G. M. Luke, B. J. Sternlieb, J. H. Brewer, J. F. Carolan et al., Phys. Rev. Lett. 62, 2317 (1989).

[64] Y. J. Uemura, A. Keren, L. P. Le, G. M. Luke, B. J. Sternlieb, W. D. Wu, J. H. Brewer, R. L. Whetten, S. M. Huang, S. Lin, R. B. Kaner, F. Diederich, S. Donovan, G. Grüner, and K. Holczer, Nature (London) 352, 605 (1991).

[65] Y. J. Uemura, J. Phys. Condens. Matter 16, S4515 (2004). [66] Y. J. Uemura, Physica (Amsterdam) 404B, 3195 (2009). 